\title{
Micro Camera Augmented Endoscopic Instruments: Towards Superhuman Performance in Remote Surgical Cutting
}

\author{
C. Staub ${ }^{1}$, C. Lenz ${ }^{1}$, B. Jensen ${ }^{1}$, S. Can ${ }^{1}$, and A. Knoll ${ }^{1}$ \\ R. Bauernschmitt ${ }^{2}$
}

\begin{abstract}
This paper introduces a new method for remote surgical cutting by providing haptic guidance along a trajectory that is measured in-situ through a miniature, tool-tip mounted endoscopic camera. By directly augmenting the instrument with a camera, instead of relying on an additional conventional endoscope, the approach becomes independent from inherent calibration uncertainties of the telemanipulation system, other than the micro camera itself, and from registration of pre- and intra-operative surgical data. We calculate a smooth cut path with corresponding scalpel orientation to guide the user towards the optimal trajectory. Experiments are conducted with passive and active haptic virtual fixtures. During active fixturing the optimal forward velocity is calculated to follow the path. The results indicate an improvement in terms of position accuracy, blade orientation, and forward velocity.
\end{abstract}

Index Terms-haptic guidance, surgical cutting, medical robotics, telemanipulation

\section{INTRODUCTION}

Haptic feedback is considered to play an important role in robotic surgery, relieving the surgeon during tiring and error-prone tasks, preventing force-induced tissue damages, and contributing to a better performance. A distinction is made between the direct feedback of forces, measured by means of dedicated force sensors [1] and the feedback of artificially generated forces. The later group includes virtual fixtures (VF), originally defined by Rosenberg [2] as an "abstract perceptual sensory information overlaid on top of reflected sensory feedback from a remote environment". While the feedback signal can also be given visually or auditory we focus on haptic virtual fixtures (HVF). Haptic constraints capitalize on the accuracy of robotic systems, enhancing the operation speed, and reduce mental stress, while permitting the user to retain ultimate control over the system [3]. The fixtures itself can be characterized in various ways: Forbidden-region virtual fixtures, often referred to as virtual wall, restrict the movement of an end effector from entering a certain area, while guiding virtual fixtures aim to help the operator to follow an optimal path. The fixture can be implemented to take on an active or passive role. A passive fixture simply scales the user's input force to drive the operator back to a desired path, while active guidance generates forces to actively guide the operator along the predetermined path. The direction of movement is therefore

*The authors want to thank Awaiba GmbH and S. Voltz for their support and for providing us the NanEye sensors.

${ }^{1}$ Robotics and Embedded Systems, Department of Informatics, Technische Universität München, Germany staub at in.tum.de

${ }^{2}$ Isar Heart Center, Department of Cardiology and Intensive Care Medicine, Munich subject to an active influence by the system, which overlays the user input.

An inherent problem that all types of HVF share is the generation of the constraint information itself. More precisely, the question of how to obtain an accurate geometrical distance measurement between the end effector and a potential fixture, which defines the force vector, and how to relate this vector with the coordinate frame of the haptic device is essential and significantly affects the quality of the haptic feedback. In remote surgery, geometrical measurements can be acquired visual e.g., in-situ by means of the endoscopic camera, or by establishing correspondences between preoperative data (e.g., CT scans [4]) and the endoscopic image stream. Although observing the objective defining the virtual fixture visually has the potential to cope with the dynamic nature of the situs, it strongly suffers from inherent registration inaccuracies. The registration of models with the patient's anatomy will introduce errors that decrease the measurement accuracy, especially in the case of deformable soft tissue registration. For instance, fixtures proposed for cardiac surgery can only roughly delineate critical regions of larger scales [5]. In the case of moving fixtures, as used during beating heart surgery (e.g., [6], [7]), motion prediction errors impede an accurate measurement. In telesurgery, many error sources of the robotic system typically contribute to a comparably bad overall calibration [8]. With respect to the experimental system utilized for this work (cf. Sec. III), these error sources include primarily the absolute accuracy and mounting angles of the robots itself, poorly measurable transformations between the individual robots, and the flexibility of the surgical instrument's carbon fiber shaft. All uncertainties mentioned, sum up if the endoscopic camera is used to derive a vision-based fixture for a surgical tool that is mounted on a different manipulator than the camera. For our system this measurement error is already about $0.5 \mathrm{~cm}$ in average and therefore does not meet the requirements for haptic guided tasks in $\mathrm{mm}$-scale, such as surgical cutting [8]. On the other hand, cooperative control systems, such as the JHU steady hand robot, have demonstrated the feasible achievable accuracy if the tool to be controlled and the camera used for fixture generation are combined in a handin-eye setup, eliminating most error sources other than the camera itself [9].

To our knowledge, these are the first reported results of tackling the precise generation of vision-based virtual haptic fixtures on a master/slave type telemanipulation system by means of micro-camera augmented instruments, applied to 


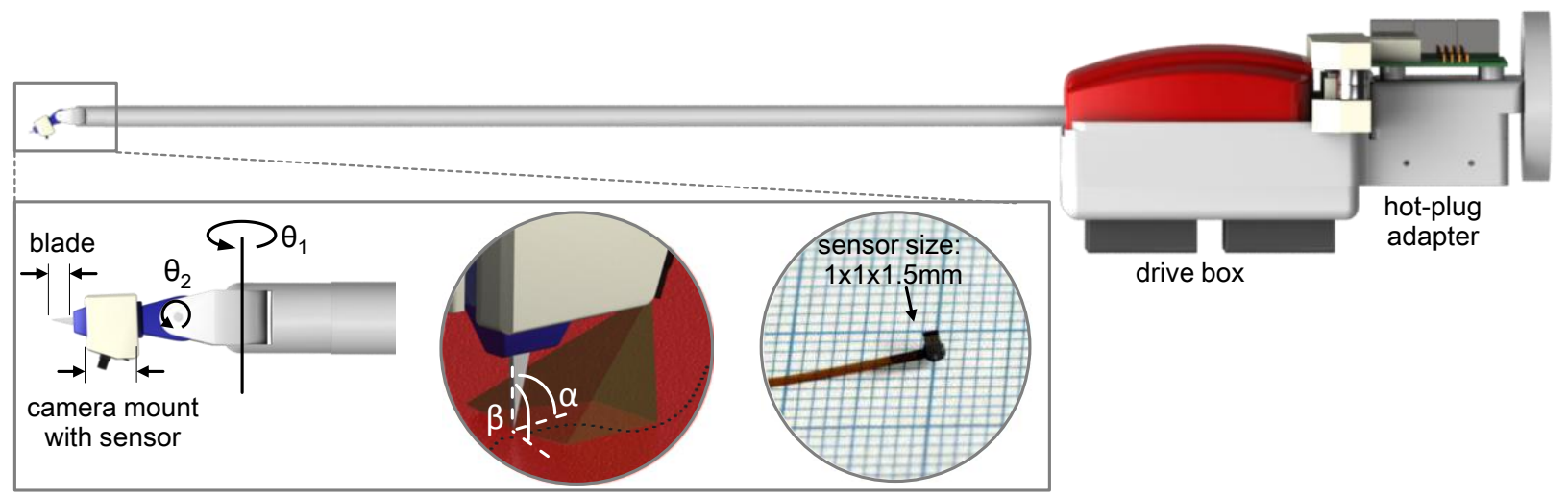

Fig. 1. Camera Augmented Surgical Instrument: The end effector consists of wrist and hand. The axis of rotation of the wrist $\theta_{1}$ is arranged perpendicular to the rotation $\theta_{2}$ of the finger. In combination with robotic arm, which carries the instrument, 6 DoF motion is restored inside the patient despite the restrictions of the trocar point. The micro camera is fixed on a dedicated mount, which can be plugged onto the distal end of the instrument. The actual blade length is about $2.5 \mathrm{~mm}$, the mount size is approximately $0.4 \times 0.5 \times 0.5 \mathrm{~cm}$. Different versions of the mount come with different bevel angles of the mounting surface in order to experiment with the provided viewing angle (see greenish indicated FOV). A four-conductor flat ribbon cable is connected to the hot-plug system of the instrument.

the delicate task of surgical cutting. Our guidance process consists of (a) the minimization between actual blade position and optimal trajectory and (b) the orientation of the blade that is automatically optimized with respect to the cut direction in order to prevent tissue damage. In this way the approach enjoys similar advantages as traditional visual-servoing techniques, but becomes applicable to delegate processes that are not adequately observable by the conventional endoscopic camera e.g., because the instrument itself obscures the field of view or small structures such as the blade with a length of only $2.5 \mathrm{~mm}$ is difficult to identify in endoscopic images. The property of the introduced method to determine the optimal motion velocity is in particular interesting for active guidance. In this context, [10] investigated the impact of speed characteristics during different actively guided tasks in an admittance-type setting. Our approach goes beyond this in the sense of choosing the optimal forward velocity within the path following task.

\section{MATERiAls AND MethodS}

In this section we define the problem of surgical cutting, derive a method that allows us to precisely follow an arbitrary cut line with optimal velocity while automatically adjusting the blade orientation and finally investigate how to constrain the surgeon's motion to this path.

\section{A. Task Description}

We use a commercially available Intuitive Snap-fit ${ }^{\mathrm{TM}}$ scalpel, equipped with a $15^{\circ}$ blade, to perform cutting. The instrument is originally deployed with the daVinci ${ }^{\mathrm{TM}}$ system [11]. The distal end of the instrument is augmented with a micro-camera, which observes the cut path area immediately preceding (cf. Fig. 1). The camera is mounted stationary with respect to the blade, capable of observing both the cut path and the blade. Thus, the alignment error can be measured in pixel units, since the camera is axis aligned with the blade. Since the camera moves with the blade, instrument movements are perceived as counter-movement to the observed surface. We assume the path to be cut to be visually identifiable (e.g., a vessel or a unique anatomical structure). The instrument is mounted on one of the slave manipulators of our experimental remote surgery system (cf. Sec. III). The operator controls the instrument by means of a 6 DoF master-side haptic device (Sensable Phantom ${ }^{\mathrm{TM}}$ ), whereas movements of the input stylus are directly mapped onto the surgical tool. The cut task itself can be decomposed into two subtasks. The first task involves augmenting the manual cut performed by the operator with haptic feedback in order to minimize the deviation of the scalpel with respect to the optimal trajectory. The second task ensures the blade orientation is aligned with the current cut direction to prevent tissue fissures. This orientation is perpendicular to the tangent of the trajectory as long as moving along the optimal path. When holding the scalpel directly in hand, as in conventional surgery, a self-alignment torque of the blade facilitates guidance by damping unwanted angular motions due to the contact between tissue and blade sides. In telesurgery, this contact force can usually not be measured and fed back to the operator, thus limiting blade alignment to visualmotor mapping of the operator. Simultaneously, the system keeps the instrument wrist automatically perpendicular to the surface (Fig. 1, angle $\beta$ ), while the steepness of the cutting angle is kept at a predefined constant value (Fig. 1, angle $\alpha)$.

\section{B. Cut Line Following}

For now, we assume the cut line given as a plane parametric curve equation

$$
\mathbf{c}(r) \equiv(x(r) \quad y(r))^{\mathrm{T}}, \quad r \in[0,1]
$$

Let $\mathbf{q}=\left(q_{x}, q_{y}\right)$ be the tip of the blade. We define $\mathbf{c}(\hat{r}(\mathbf{q}))$ as the curve point with minimal distance from the blade as

$$
\|\mathbf{c}(\hat{r}(\mathbf{q}))-\mathbf{q}\|=\min _{r \in[0,1]}\|\mathbf{c}(r)-\mathbf{q}\|
$$




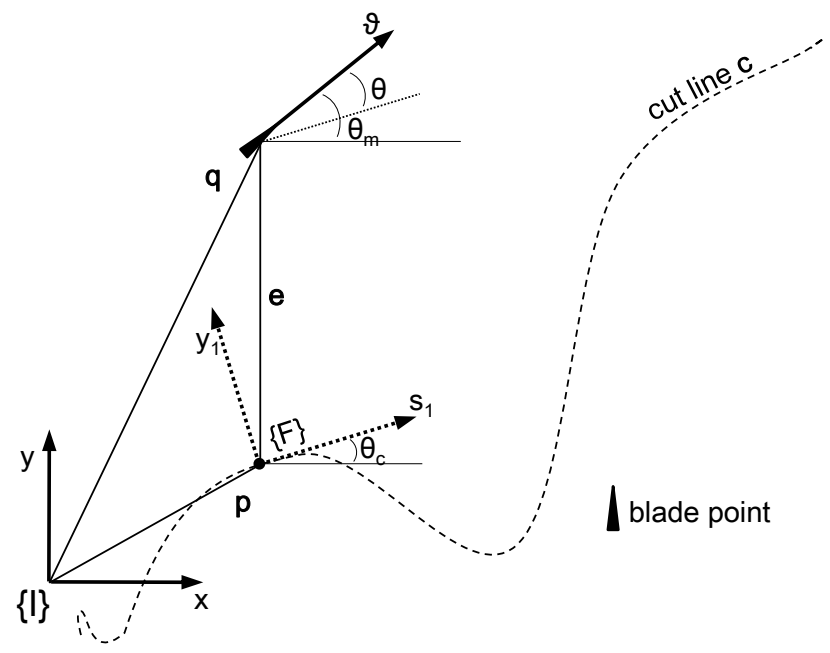

Fig. 2. Guided cutting: The position of the blade $\mathbf{q}$ is expressed in frame $\{I\}$ and a Frenet frame $\{F\}$ rooted in the tangent space of the path. The angle $\theta_{m}$ needs to be reduced to zero to guide the scalpel back to the optimal trajectory.

As long as the blade follows the optimal trajectory the direction of the tool tip at that point is the normalized tangent direction $\theta_{c}$.

$$
\begin{aligned}
\mathbf{t}(\mathbf{q}) & =\left.\frac{d}{d t} \mathbf{c}(r)\right|_{r=\hat{r}(\mathbf{q})} \\
\theta_{c}(\mathbf{q}) & =\frac{\mathbf{t}(\mathbf{q})}{\|\mathbf{t}(\mathbf{q})\|}
\end{aligned}
$$

Once the tool tip differs from the optimal trajectory, we can define a Cartesian error vector e as

$$
\mathbf{e}(\mathbf{q})=\mathbf{q}-\mathbf{c}(\hat{r}(\mathbf{q}))=\mathbf{q}-\mathbf{p}
$$

In this case, we want to guide the user smoothly back to the optimum trajectory, taking the current forward motion and the blade orientation into account. It is not desirable to minimize e rapidly, without taking the blade orientation into account. In terms of smooth guidance, it is desirable to calculate a path that converges as fast as possible to the optimum trajectory while preventing rapid changes of direction. Please note that attaching the micro camera at the instrument tip allows us to treat the path following problem in the frame of the haptic input device, rather than in a calibration-dependent, error-prone world frame. Instead of the global tool position we can directly utilize the input of the haptic device, which guides the instrument.

For a given cut path c we obtain a smooth trajectory if the lateral error vector $\mathbf{e}$ is minimized and the blade orientation equals $\theta_{c}$ (cf. Fig. 2). The development of constraints on the blade motion is comparable to the nonholonomic constraints of differential-driven mobile robots: the motion is governed by a forward velocity and an angular velocity, while any lateral movements are to be avoided. We adapt a solution presented in [12] and treat our problem in terms of a SerretFrenet frame $\{F\}$ that moves tangential along $\mathbf{c}$ and reflects a virtual target frame. As we follow the curve along a given direction, we consider the signed curvilinear abscissa of $\{F\}$, denoted as $s_{1}$, instead of the undirected tangent $\mathbf{t}(\mathbf{q})$ in (3). Now, let ${ }^{I} \mathbf{q}$ be the current blade position on the tissue with ${ }^{I} \mathbf{q} \neq{ }^{I} \mathbf{p}$, where ${ }^{I} \mathbf{p}=\mathbf{c}\left(\hat{r}\left({ }^{I} \mathbf{q}\right)\right)$ is the curve point with minimal error $\mathbf{e}$ as defined above. The blade point can be described in the inertial reference frame $\{I\}$ as ${ }^{I} \mathbf{q}=\left[\begin{array}{lll}{ }^{I} q_{x} & { }^{I} q_{y} & 0\end{array}\right]^{\mathrm{T}}$ and in $\{F\}$ as ${ }^{F} \mathbf{q}=\mathbf{e}=\left[\begin{array}{lll}{ }^{F} q_{s_{1}} & { }^{F} q_{y_{1}} & 0\end{array}\right]^{\mathrm{T}}$. Equivalently, $\mathbf{p}$ is given in $\{I\}$ as ${ }^{I} \mathbf{p}=\left[\begin{array}{lll}{ }^{I} p_{x} & { }^{I} p_{y} & 0\end{array}\right]^{\mathrm{T}}$ and in $F$ always as ${ }^{F} \mathbf{p}=\mathbf{0}_{[3 \times 1]}$. Further, let ${ }_{F}^{I} R$ be the rotation matrix from $\{I\}$ to $\{F\}$, parameterized by the angle $\theta_{c}$ of the curvilinear abscissa $s$ with respect to ${ }^{I} \mathbf{e}_{x}$ and ${ }_{I}^{F} R={ }_{F}^{I} R^{-1}$ the reverse rotation respectively. The tangential velocity in ${ }^{F} \mathbf{p}$ is denoted as $\dot{s}$. The angular velocity of $\theta_{c}$ is then defined by

$$
\omega_{c}=\dot{\theta}_{c}=\kappa\left(\mathbf{c}\left(\hat{r}\left({ }^{F} \mathbf{q}\right)\right)\right) \dot{s}
$$

with $\kappa(\cdot)$ being the curvature of the trajectory.

According to these definitions, the velocities of both points $\mathbf{p}$ and $\mathbf{q}$ can be expressed straightforward in both frames

$$
\begin{aligned}
{ }^{F} \dot{\mathbf{p}} & ={ }_{F}{ }_{F}{ }^{I} \dot{\mathbf{p}} \\
& =\left[\begin{array}{lll}
\dot{s} & 0 & 0
\end{array}\right]^{\mathrm{T}}
\end{aligned}
$$

The velocity of $\mathbf{q}$ in $\{I\}$ is given by

$$
\begin{aligned}
{ }^{I} \dot{\mathbf{q}} & =\left[\begin{array}{lll}
{ }^{I} \dot{q}_{x} & { }^{I} \dot{q}_{y} & 0
\end{array}\right]^{\mathrm{T}} \\
& ={ }^{I} \dot{\mathbf{p}}+{ }_{I}^{F} R \dot{\mathbf{e}}+{ }_{I}^{F} R\left(\omega_{c} \times \mathbf{e}\right)
\end{aligned}
$$

with e being our error vector from $\mathbf{p}$ to $\mathbf{q}$, characterizing the deviation of the blade from the optimal trajectory. Now, the velocity of ${ }^{I} \mathbf{q}$ can be expressed in $\{F\}$ by multiplying both sides with ${ }_{F}^{I} R$

$$
\begin{aligned}
{ }^{F} \dot{\mathbf{q}} & ={ }_{F}^{I} R^{I} \dot{\mathbf{q}} \\
& ={ }^{F} \dot{\mathbf{p}}+\dot{\mathbf{e}}+\left(\omega_{c} \times \mathbf{e}\right)
\end{aligned}
$$

The relation

$$
\begin{aligned}
\omega_{c} \times \mathbf{e} & =\left[\begin{array}{c}
0 \\
0 \\
\kappa(s) \dot{s}
\end{array}\right] \times\left[\begin{array}{c}
F \\
{ }^{F} q_{1} \\
{ }^{F} q_{y_{1}} \\
0
\end{array}\right] \\
& =\left[\begin{array}{c}
-\kappa(s) \dot{s} \cdot{ }^{F} q_{y_{1}} \\
\kappa(s) \dot{s} \cdot{ }^{F} q_{s_{1}} \\
0
\end{array}\right]
\end{aligned}
$$

yields to an expression of (9) as

$$
{ }^{F} \dot{\mathbf{q}}=\left[\begin{array}{c}
\dot{s}\left(1-\kappa(s)^{F} q_{y_{1}}\right)+{ }^{F} \dot{q}_{s_{1}} \\
{ }^{F} q_{y_{1}}+\kappa(s) \dot{s} \cdot{ }^{F} q_{s_{1}} \\
0
\end{array}\right]
$$

which is solved for ${ }^{F} \dot{q}_{s_{1}}$ and ${ }^{F} \dot{q}_{y_{1}}$ :

$$
\begin{aligned}
& { }^{F} \dot{q}_{s_{1}}=\left[\begin{array}{ll}
\cos \theta_{c} & \sin \theta_{c}
\end{array}\right]\left[\begin{array}{c}
{ }^{I} \dot{q}_{x} \\
{ }^{I} \dot{q}_{y}
\end{array}\right]-\dot{s}\left(1-\kappa(s) \cdot{ }^{F} q_{y_{1}}\right)(12 \\
& { }^{F} \dot{q}_{y_{1}}=\left[\begin{array}{ll}
-\sin \theta_{c} & \cos \theta_{c}
\end{array}\right]\left[\begin{array}{c}
{ }^{I} \dot{q}_{x} \\
{ }^{I} \dot{q}_{y}
\end{array}\right]-\kappa(s) \dot{s}^{F} q_{s_{1}}
\end{aligned}
$$

Applying the current linear velocity $v$ of the tip (which is a combination of the velocity applied by the user and the calculated forward velocity), the rotation angle $\theta_{m}$ of the 
blade in $\{I\}$, the respective angular velocity $\omega=\omega_{m}=\dot{\theta}_{m}$, substituting

$$
\left[\begin{array}{c}
{ }^{I} \dot{q}_{x} \\
{ }^{I} \dot{q}_{y}
\end{array}\right]=v\left[\begin{array}{c}
\cos \theta_{m} \\
\sin \theta_{m}
\end{array}\right]
$$

in (12) and (13), and applying the mathematical rules for $\theta=\theta_{m}-\theta_{c}$ :

$$
\begin{aligned}
\sin \theta & =\sin \theta_{m} \cos \theta_{c}+\cos \theta_{m} \sin \theta_{c} \\
\cos \theta & =\cos \theta_{m} \cos \theta_{c}+\sin \theta_{m} \sin \theta_{c}
\end{aligned}
$$

yields to our final instantaneous kinematic model of the blade tip in $\{F\}$

$$
\begin{gathered}
{ }^{F} \dot{q}_{s_{1}}=-\dot{s}\left(1-\kappa(s) \cdot{ }^{F} q_{y_{1}}\right)+v \cos \theta \\
{ }^{F} \dot{q}_{y_{1}}=-\kappa(s) \dot{s} \cdot{ }^{F} q_{s_{1}}+v \sin \theta \\
\dot{\theta}=\omega-\kappa(s) \dot{s}
\end{gathered}
$$

If parameters ${ }^{F} q_{s_{1}},{ }^{F} q_{y_{1}}$ and $\theta$ are driven towards zero, the blade optimally follows the trajectory. During passive fixturing, we are interested in optimizing only the angular velocity $\omega$ of the blade

$$
\omega=\dot{\theta}_{m}+\kappa(s) \dot{s}
$$

Therefore, we follow [13] and choose $\dot{\theta}$ and $\dot{s}$

$$
\begin{aligned}
\dot{\theta} & =\dot{\delta}-\gamma \cdot{ }^{F} q_{y_{1}} \cdot v \frac{\sin \theta-\sin \delta}{\theta-\delta}-k_{2}(\theta-\delta) \\
\dot{s} & =v \cos \theta+k_{1} \cdot{ }^{F} q_{s_{1}}
\end{aligned}
$$

with scaling factors $k_{1}, k_{2}>0$. The function $\delta$ shapes the transition between desired and current trajectory during the path approach to zero and was chosen as $\delta=k_{3} \tanh y_{1}^{a}$, with $k_{3}>0$ and $a$ being and odd exponent. The forward velocity is manually controlled by the operator.

During active fixturing, we can also control an optimal forward velocity, according to

$$
v= \begin{cases}c V_{\max } & , \text { if } V_{1} \geq \varepsilon \\ \frac{1}{1+|\kappa(s)|} V_{\max } & , \text { if } V_{1}<\varepsilon\end{cases}
$$

where threshold $c<1$ decelerates the movement when exceeding a path error, and $V_{\max }$ is the maximum defined forward velocity of the blade. The control law guarantees the maximum forward velocity with respect to the current curvature. According to [12], we choose for the control law the Lyapunov candidate function

$$
V_{1}=\frac{1}{2}\left({ }^{F}{q_{s_{1}}}^{2}+{ }^{F} q_{y_{1}}{ }^{2}\right)+\frac{1}{2 \gamma}\left(\theta-\delta\left({ }^{F} q_{y 1}, v\right)\right)^{2}
$$

\section{Commanded Input Motion and Feedback Generation}

The tool tip mounted camera allows us to evade errorprone intrinsic system uncertainties. The resulting error in pixel units corresponds to the instrument frame, which in turn is aligned with the frame of the haptic device, in order to allow an intuitive hand-eye coordination during teleoperation. A deviation between tool-tip and desired trajectory, measured in image space, can thus directly be coupled to the generation for haptic guidance.
Recalling the last section, we need to update the current blade position $\mathbf{q}$ as well as the angle $\theta$ in every time step and receive the corresponding cut direction update $\omega$ along with a forward velocity $v$. The input can directly be derived from the motion of the haptic device. In order to cancel involuntary jerky movements to the patient-side robots we first filter the user's input motion. Human hand tremor e.g. overlays voluntary motion at the haptic input devices with a roughly sinusoidal approximate motion [14]. In order to suppress this signal it can be estimated on-line by a weightedfrequency Fourier linear combiner [15]. The algorithm adapts to the dominant frequency of a quasi-periodic signal over time, whereas both frequency and amplitude are unknown. The adaption to the input signal is obtained by changing amplitude and frequency weights of a dynamic truncated Fourier series model via gradient descent optimization.

The generation of the feedback signals can be divided into a general part, limiting the dynamic behaviour of the haptic input device with an artificial non-linear damping to prevent rapid changes in direction and high input velocities during the cut, and a task specific part, which generates the actual guiding force. The resistance of the general part is realized as damping of the commanded stylus movement. It is implemented by means of an exponential envelope $s_{d}=\left(1-\exp \left(-a l^{2}\right)\right) \cdot f_{\max }$ that restricts the maximum speed of the stylus, where $a$ is related to the stiffness of the system, $f_{\max }$ is the maximum force value, and $l$ being the distance between the current and the previous position $l=\mathbf{q}_{t-1}-\mathbf{q}$. The applied force vector is then

$$
\mathbf{f}_{d}=\frac{\mathbf{q}_{t-1}-\mathbf{q}}{\left\|\mathbf{q}_{t-1}-\mathbf{q}\right\|} \cdot s_{d}
$$

The application of an exponential function ensures a smooth force progression, as sudden force changes usually lead to unpredictable vibrations at the end effector, while being still capable of simulating high stiffness. The VF is realized on a proxy-based implementation, where the proxy represents the calculated optimal position and the master servos to the proxy. The master is controlled by

$$
\mathbf{f}_{g}=k_{p}(\mathbf{p}-\mathbf{q})+k_{d}(\dot{\mathbf{p}}-\dot{\mathbf{q}})
$$

where $k_{p}$ and $k_{d}$ are the proportional and derivative gains respectively. Our haptic device has a sampling rate of $1000 \mathrm{~Hz}$, which is considered to be the lower limit for jerk-free rendering of rigid contacts. The mismatch between camera frame rate and haptic loop cycle is compensated by means of a Kalman filter. While the position of the blade is predicted in between the camera frames, the prediction is corrected as soon as vision-based fixtures can be derived. This effectively suppresses an oscillation of the system during cutting. The final force is thus composed of

$$
\mathbf{f}=\mathbf{f}_{d}+\mathbf{f}_{g}
$$

\section{IMPLEMENTATION}

The algorithms described above were implemented on our system for robot-assisted minimally invasive surgery. We briefly describe the setup, more details can be found in 
[1]. The master console of the telepresence system offers surgeons the ability to operate the slave-side robots via two PHANToM ${ }^{\mathrm{TM}}$ Premium 1.5 haptic devices. The force feedback capabilities of the haptic devices can be used to exert forces in translational directions (3 DoF). A 3D display visualizes the data that is acquired by a stereoscopic endoscope. The slave manipulator of the system consists of four ceiling mounted Mitsubishi $6 \mathrm{SL}^{\mathrm{TM}}$ manipulators, providing 6 DoF each. A magnetic coupling mechanism, which also establishes all necessary electrical connections via spring contacts (e.g., for the instruments servos), carries exchangeable minimally invasive instruments.

The micro camera for guided cutting is a prototype of the Awaiba NanEye $2 \mathrm{~B}^{\mathrm{TM}}$, operating at 30fps [16]. To our knowledge, the system-on-chip sensor is currently the smallest CMOS camera with a total size of approximately $(1 \times 1 \times 1.5) \mathrm{mm}$ including a lens that provides $90^{\circ}$ field of view and a resolution of $250 \times 250$ pixels. In order to attach the micro camera at the distal end of the instrument we developed a laser sintered mount (cf. Fig. 1). The sensor is orientated such that the field of view begins just behind the scalpel tip and covers the widest possible view of the cutting path.

\section{EXPERIMENTAL EVALUATION}

An evaluation to prove the feasibility and power of our approach was conducted within the simulation environment of our system and first experiments were transfered to hardware. In order to obtain real-world conditions for the simulation, we artificially delay the image-based fixture generation to 25 frames per second. This is the lower limit of the NanEye ${ }^{\mathrm{TM}}$ sensor. The delay introduces a mismatch between haptic loop and image processing which, if not compensated, can result in system oscillations (cf. Sec. II-C). The experiments have been conducted with the master console presented above, providing an elbow rest for the users to position themselves comfortably and without physical exertion. The screen is used to either display the video stream of the micro camera itself or the simulation. The cut task was performed in $2 \mathrm{D}$ space, hence the user could not alter the height between tool and surface or change penetration depth. The restriction was implemented as VF on both master and slave. During guidance experiments, a HVF with high stiffness on the master-side prevents the user from penetrating a plane, while the master neglects commands perpendicular to the cut plane.

The experiments were conducted with 10 untrained subjects. Each of them was instructed to move along the path quickly as possible without sacrificing accuracy, considering both factors equally important. After at least one familiarizing initial trial the subjects repeated each experiment two times. All experiments were randomly performed, testing four levels of guidance.

1) Experiment 1: Manual control. During this experiment the user has to follow the trajectory without any type of guidance. He simultaneously needs to minimize the error to the trajectory, while taking care of the blade orientation. The orientation can be adjusted by rotating the stylus of the haptic input device.

2) Experiment 2: Passive guidance without blade angle optimization. During this experiment, the user is passively guided along the trajectory. The error between current blade position and optimal path is calculated and fed back as haptic signal. The blade angle is automatically set according to the current forward direction. To cope with the $1 \mathrm{kHz}$ rate of the haptic device the values are smoothed over time by means of an exponential time series filter [17] to avoid permanent angle adjustments caused by small movements.

3) Experiment 3: Passive guidance, optimized blade angle During this experiment, the user is passively guided along the trajectory. The error between current blade position and optimal path is calculated and fed back as haptic signal. The blade angle is automatically set according to the proposed method in Sec. II-B.

4) Experiment 4: Active guidance, optimized blade angle During this experiment, the user is actively guided along the trajectory. The forward motion of the tool is calculated according to the proposed method in Sec. II-B, as is the blade angle. The maximum velocity depends on the parameterization.

During the experiments, the control computer recorded the completion time and the movement of the virtual instrument. The user could choose an arbitrary start position at the beginning of the curve. We calculated the deviation of the blade tip from the optimal path in pixels. The blade orientation was measured with respect to the current forward direction, referred to as blade alignment. This parameter is more meaningful than the alignment error with respect to the tangent of the given cut path, since it characterizes the smoothness of the cut and indicates tissue fissures. Figure 4 shows plots of the recorded trajectories for each subject. The corresponding absolute mean position and angle alignment errors (black colored), with standard deviation (red colored), are calculated pixel wise. Table I shows the overall errors, averaged over all subjects and all trajectories with corresponding completion times. The overall errors should not be overstated: the relatively small overall mean position error of $7 p x$ during unguided cutting sounds like a good result, but a look at the plots reveals a highly irregular motion. This effect is also evident in the corresponding standard deviation, which is doubled compared to guided cutting and even quadrupled compared to our guidance approach. Furthermore the average blade alignment error of $15^{\circ}$ would make a cut difficult in practice, whereas the amount of jitter is distinctive. The angle is constantly adjusted by the user. However, as mentioned above, a smooth movement can be considered as a key factor for successful cutting. A look at the trajectory plots and positioning errors shows a strong effect of haptic guidance, whereas the quality with respect to the positioning error can be considered equally for all guidance types. During passive fixturing, an error increase can be observed at the turning point of the curve, between pixels 200-300. Active guidance (experiment 4) could minimize this effect as the input velocity is adapted and user's motion decelerates. 

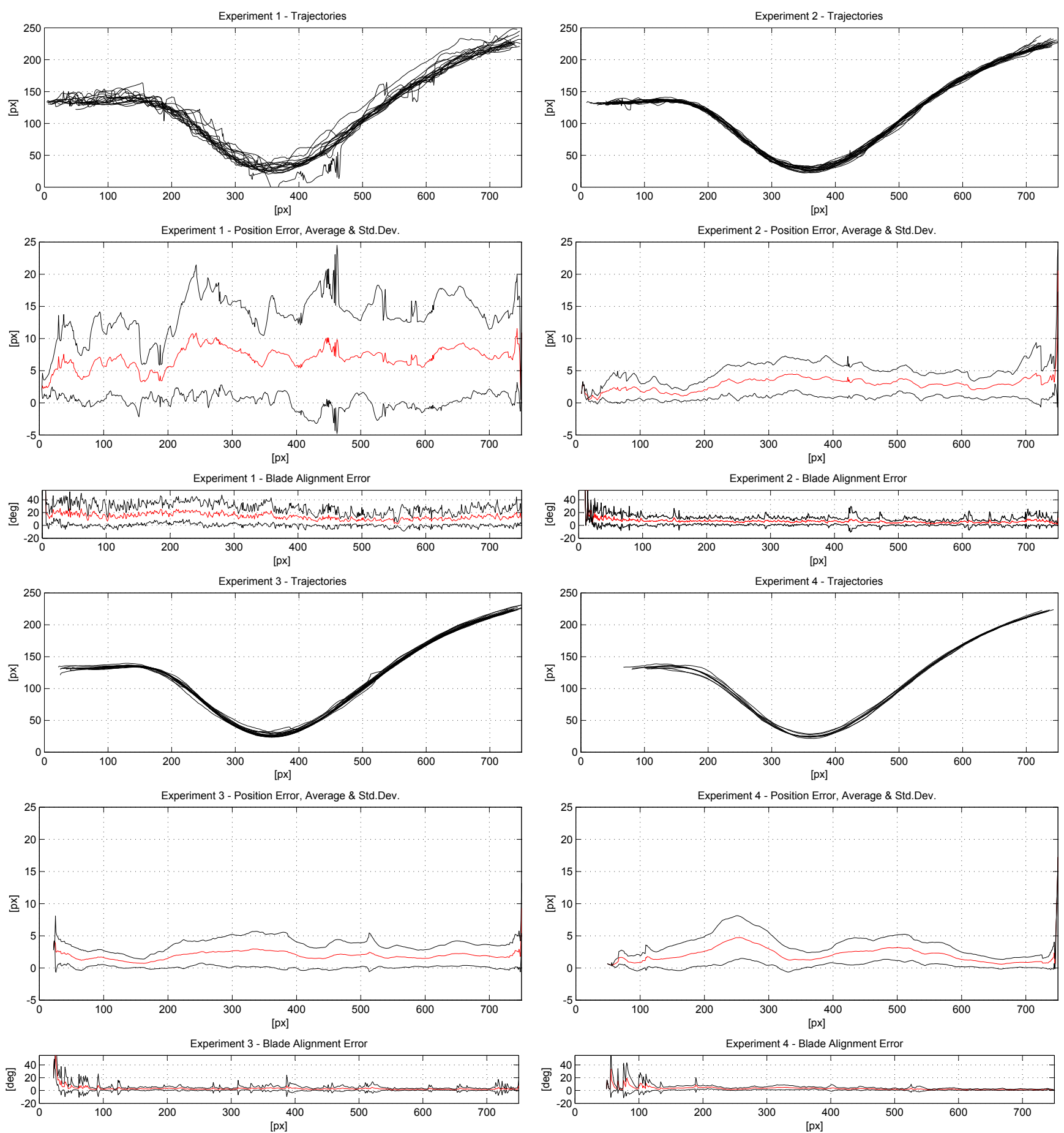

Fig. 3. Experimental results for 10 subjects for all four experiments: manual control (Experiment 1), passive guidance without blade angle optimization (Experiments 2), passive guidance with optimized blade angle (Experiment 3), and active guidance with optimized blade angle (Experiment 4). Shown are the recorded trajectories, with mean positioning error (red) and standard deviation (black), and the blade alignment error with respect to the forward motion of the instrument, also with mean position (red) and and standard deviation (black).

Curve sections undergoing an acceleration exhibit a higher deviation than the remaining sections of the curve, e.g., pixels 200-250 and 420-570. A look at the blade alignment errors suggest that automated blade orientation greatly improves the error. Our proposed method outperforms experiment 2 by a factor of two and the manual alignment by a factor of five. For experiment 2 the blade orientation is governed by a time-dependent filter. The chosen timing of the filter was a trade-off between reaction time, in order to quickly adapt to new angles, and smoothing to avoid jitter. Although the angle alignment of our method works equally well for passive and active guidance (cf. Table I), the combination with active fixturing seems to be advantageous in terms of jitter reduction. The effect is reflected in the error plot 
TABLE I

ERRORS AND EXECUTION TIME

\begin{tabular}{ccccccc}
\hline & \multicolumn{2}{c}{ pos. error $[\mathrm{px}]$} & \multicolumn{2}{c}{ angle error [deg] } & \multicolumn{2}{c}{ execution time [s] } \\
\cline { 2 - 7 } Exp. & mean & std.dev. & mean & std.dev. & mean & std.dev. \\
\hline 1 & 7.00 & 1.97 & 15.79 & 4.67 & 20.01 & 5.38 \\
2 & 2.92 & 0.82 & 6.69 & 3.20 & 14.90 & 4.60 \\
3 & 1.92 & 0.54 & 3.15 & 3.18 & 7.87 & 1.45 \\
4 & 2.07 & 0.74 & 3.14 & 3.47 & 5.83 & 0.97 \\
\hline
\end{tabular}

of experiments 3 and 4, whereas experiment 4 shows less peaks in the alignment error. Task completion time could be halved, whereas active guidance could achieve only a small advantage.

During the experiments, most subjects found it difficult and exhausting to follow a path while simultaneously coping with manual blade alignment. The missing self-alignment force during telemanipulation could however be compensated by an automated alignment of the scalpel. For active fixturing, both the lateral and the angular error were decreased, while task completion time was substantially faster. One might argue that a fast execution might not be relevant, however, our method guides the user in the sense of an optimal forward velocity. In doing so the method ensures velocities not exceeding a certain limit on critical path sections, i.e. those with increased curvature. Promising experiments have also been performed on hardware. At that, the accuracy is currently limited by the play of the servo motors and bowdn-wires that actuate the last three degrees of freedom of the instrument (cf. Fig. 1). Mainly the angular accuracy is affected, while the lateral position can be controlled precisely.

\section{Conclusion And Outlook}

A new method for remote surgical cutting by providing haptic guidance has been proposed. The approach becomes independent from calibration uncertainties of typical remote surgery robots by directly augmenting surgical tools with a micro camera. A smooth cut path with corresponding scalpel orientation to guide the user toward the optimal trajectory was calculated, using both passive and active haptic virtual fixtures. The approach could confirm its advantages in terms of accuracy and execution time during experiments carried out in a realistic environment.

Future work includes the extension of the task to the third dimension. Depth perception can be integrated by either a stereoscopic setup of the micro cameras, by means of the instrument's strain-gauge sensors, or by fusing both modalities. Especially the integration of force data seems to be promising, as it is available at a rate of $1 \mathrm{kHz}$. This allows for a fast adaption of the cutting depth, which is indispensable to avoid tissue fissures. A more advanced haptic device, providing torque force feedback, could potentially support the angular orientation by actuating the stylus' self rotation. The calculated angular velocity therewith becomes a virtual self-alignment torque, which can be fed back to the operator. Directly measuring the blade's torque in-situ and feeding it back through teleoperation would allow a combination with the angular virtual fixture. However, measuring this angular force is quite challenging and the authors are not aware of a suitable solution.

\section{REFERENCES}

[1] H. Mayer, I. Nagy, A. Knoll, E. Braun, R. Bauernschmitt, and R. Lange, "Haptic feedback in a telepresence system for endoscopic heart surgery," MIT PRESENCE: Teleoperators and Virtual Environments, vol. 16, no. 5, pp. 459-470, 2007.

[2] L. Rosenberg, "Virtual fixtures: Perceptual tools for telerobotic manipulation," in IEEE Virtual Reality Annual International Symposium, 1993, pp. 76-82.

[3] C. Passenberg, A. Peer, and M. Buss, "A survey of environment, operator-, and task-adapted controllers for teleoperation systems," Mechatronics, vol. 20, no. 7, pp. 787 - 801, 2010.

[4] M. Li and R. Taylor, "Spatial motion constraints in medical robot using virtual fixtures generated by anatomy," in Proceedings of the IEEE International Conference of Robotics and Automation, vol. 2, 2004, pp. $1270-1275$.

[5] S. Park, R. D. Howe, and D. F. Torchiana, "Virtual fixtures for robotic cardiac surgery," in MICCAI, 2001, pp. 1419-1420.

[6] J. Ren, R. Patel, K. McIsaac, G. Guiraudon, and T. Peters, "Dynamic 3-d virtual fixtures for minimally invasive beating heart procedures," IEEE Transactions on Medical Imaging, vol. 27, no. 8, pp. 1061 1070, 2008.

[7] T. L. Gibo, L. N. Verner, D. D. Yuh, and A. M. Okamura, "Design considerations and human-machine performance of moving virtual fixtures," in Proceedings of the IEEE International Conference of Robotics and Automation, 2009, pp. 671-676.

[8] C. Staub, G. Panin, A. Knoll, and R. Bauernschmitt, "Visual instrument guidance in minimaly invasive robot surgery," International Journal of Advances in Life Sciences, vol. 2, no. 3\&4, pp. 103-114, 2010.

[9] A. Bettini, P. Marayong, S. Lang, A. M. Okamura, and G. D. Hager, "Vision-assisted control for manipulation using virtual fixtures," IEEE Transactions on Robotics, vol. 20, no. 6, pp. 953 - 966, 2004.

[10] D. Kragic, P. Marayong, M. Li, A. M. Okamura, and G. D. Hager, "Human-machine collaborative systems for microsurgical applications," Int. J. Rob. Res., vol. 24, no. 9, pp. 731-741, 2005.

[11] G. Guthart and J. Salisbury, "The intuitive ${ }^{\mathrm{TM}}$ telesurgery system: overview and application," Proceedings of IEEE ICRA, vol. 1, pp. 618-621, 2000.

[12] D. Soetanto, L. Lapierre, and A. Pascoal, "Adaptive, non-singular pathfollowing control of dynamic wheeled robots," in Proceedings of the IEEE Conference on Decision and Control, vol. 2, 2003, pp. 1765 1770 .

[13] A. Micaelli, A. Micaelli, C. Samson, C. Samson, and P. Icare, "Trajectory tracking for unicycle-type and two-steering-wheels mobile robots," INRIA, Research Report RR-2097, 1993.

[14] R. Elble and W. Koller, Tremor. Baltimore, USA: John Hopkins University Press, 1990.

[15] C. Riviere and N. Thakor, "Modeling and canceling tremor in humanmachine interfaces," IEEE Engineering in Medicine and Biology Magazine, vol. 15, no. 3, pp. $29-36,1996$.

[16] M. Wäny, S. Voltz, F. Gaspar, and L. Chen, "Minimal form factor digital-image sensor for endoscopic applications," in Proceedings of the SPIE: Sensors, Cameras, and Systems for Industrial/Scientific Applications, vol. 7249, San Jose, USA, 2009.

[17] P. D. Stroud, "A recursive exponential filter for time-sensitive data," Los Alamos National Laboratory, Tech. Rep. LAUR-99-5573, 1999. 